\title{
Recent advances on glass-forming systems driven far from equilibrium
}

\section{Special issue marking the completion of the Research Unit FOR 1394 'Nonlinear response to probe vitrification'}

\author{
Matthias Fuchs ${ }^{\mathrm{a}}$ \\ Fachbereich Physik, Universität Konstanz, 78457 Konstanz, Germany
}

Received 16 June 2017

Published online 10 August 2017

\begin{abstract}
The nature of the glass transition is one of the frontier questions in Statistical Physics and Materials Science. Highly cooperative structural processes develop in glass-forming melts exhibiting relaxational dynamics which is spread out over many decades in time. While considerable progress has been made in recent decades towards understanding dynamical slowing-down in quiescent systems, the interplay of glassy dynamics with external fields reveals a wealth of novel phenomena yet to be explored.

This special issue focuses on recent results obtained by the Research Unit FOR 1394 'Nonlinear response to probe vitrification' which was funded by the German Science Foundation (DFG). In the projects of the research unit, strong external fields were used in order to gain insights into the complex structural and transport phenomena at the glass transition under far-fromequilibrium conditions. This aimed inter alia to test theories of the glass transition developed for quiescent systems by pushing them beyond their original regime. Combining experimental, simulational, and theoretical efforts, the eight projects within the FOR 1394 measured and determined aspects of the nonlinear response of supercooled metallic, polymeric, and silica melts, of colloidal dispersions, and of ionic liquids. Applied fields included electric and mechanic fields, and forced active probing ('micro-rheology'), where a single probe is forced through the glass-forming host. Nonlinear stressstrain and force-velocity relations as well as nonlinear dielectric susceptibilities and conductivities were observed. While the physical manipulation of melts and glasses is interesting in its own right, especially technologically, the investigations performed by the FOR 1394 suggest to use the response to strong homogeneous and inhomogeneous fields as technique to explore on the microscopic level the cooperative mechanisms in dense melts of strongly interacting constituents.

Questions considered concern the (de-)coupling of different dynamical degrees of freedom in an external field, and the ensuing state
\end{abstract}

\footnotetext{
${ }^{\text {a }}$ e-mail: matthias.fuchs@ukn.de
} 


\begin{abstract}
diagrams. What forces are required to detach a localized probe particle from its initial environment in a supercooled liquid, in a glassy or granular system? Do metallic and colloidal glasses yield homogeneously or by strain localization under differently applied stresses? Which mechanisms determine field-dependent susceptibilities in dielectric and ionically conducting glass formers?
\end{abstract}

The central objective of the manuscripts collected in this special issue is to explore nonlinear response phenomena in glass-forming systems and to use them to gain insights into the yet open problem of the glass transition. The process of vitrification encompasses transport and relaxation processes as well as structural rearrangements of particles which exhibit strong cooperativity. The supercooling of liquids not only leads to a slowing-down of particle motion, more importantly, it enhances cooperative effects. The spectral width of relaxation processes broadens enormously and a multitude of secondary processes comes into existence beyond the terminal relaxation process. Spatial variations in the mobilities of particles increase, and the sensitivity to external fields shoots up. The growth of temporal and spatial correlations culminates in the glass transition, when a metastable amorphous solid is formed. Its mechanical, dielectric, and conductive properties cannot be understood by using formalisms developed for the description of crystalline materials. Rather one has to take into account the disorder and the memory effects, which cause the history dependence of the metastable solid. Both, the properties of supercooled liquids as well as of glasses, can be affected by external fields which cut off the slow dynamics or distort the frozenin elastic structure. An explicit, by now well understood, example is given by steady shear flow of colloidal dispersions, which melts a non-ergodic state and causes shear and normal stresses by breaking the local cages around particles. The insights gained into the non-equilibrium steady state under shear are an example that the nonlinear response of supercooled liquids to external fields is an important scientific topic for rewarding investigations of the cooperative dynamics.

The cooperative nature of the glass transition entails the still unresolved questions about the definition of structural entities which play the role of "quasi-particles" involved in plastic deformation, flow, and transport. These elementary dynamic processes accumulate during transient experiments, where the system, which initially is in equilibrium, experiences a strong external field, such as a mechanical stress or an electric bias potential. Elastoplastic models valid on mesoscopic length scales have emerged at low temperatures, which focus on localized plastic events interacting via an elastic surrounding matrix. Under external load, they accumulate into avalanchelike processes which cause macroscopic relaxation. Yet, their behavior at temperatures approaching the melting of glass, and their merging into liquid-like transport processes in supercooled liquids is not understood. Therefore, driving glass-forming systems out of equilibrium provides interesting information about these elementary processes. Another aspect deals with the understanding of the heterogeneities in dynamic or static correlations. Different nonlinear observables directly reflect specific properties of these heterogeneities. Therefore, nonlinear response experiments provide access to heterogeneities in an unique way.

Experimental, simulational, and theoretical approaches on the microscopic level are required to gain fundamental insights. The mini-reports collected in this special issue present such investigations on a number of model glass-forming systems, like colloidal dispersions, metallic alloys, ionic and molecular liquids. This multitude of different systems allows one to discern universal and system-specific properties.

A number of research themes and shared concepts unite the projects reported in this special issue. 
First, a small set of model glass formers is studied in well-controlled fields. The comparison of the different studies reveals universalities, and guides the understanding of vitrification. The relation between colloidal to metallic supercooled fluids is of interest, especially considering multi-component systems. Mixing/ alloying effects lead to a rich field of phase and kinetic behaviors, which has large technological significance. Also, the mapping between thermal systems and homogeneously driven granular systems provides insights into strongly cooperative phenomena, when forces (in micro-rheology) or stresses (in macro-rheology) are additionally applied in order to test the load-bearing structures like e.g., force chains. Micro-rheology reveals new universal features in random media, and in colloidal, molecular, and granular dense fluids, yet becomes sensitive to molecular damping mechanisms in the large-force limit. Monohydroxy alcohols, which exhibit a dominating Debye-relaxation, serve in dielectric spectroscopy as non-cooperative model systems to be contrasted with the stretched relaxation in glass formers. Both, local movements of ions (in conducting fluids) and movements of permanent dipoles (in dielectric molecular fluids), lead to fluctuating dipoles which couple to strong electric fields.

Second, insights result from comparisons of corresponding protocols of investigation. From the multitude of different protocols for applying time-dependent external fields, two simple protocols have proven useful in the investigations reported. On the one hand, large amplitude oscillatory driving opens the possibility to examine the nonlinear response as function of drive amplitude and frequency. The conductivity and dielectric but also the mechanical spectroscopy use frequency dependence to test theoretical scaling laws (describing the growth of cooperative regions), and the amplitude to determine characteristic field values (like the yield stress determining the nonlinearity in mechanical loading). These investigations begin to draw a comprehensive picture of the evolution of the nonlinear harmonic excitations in supercooled states. On the other hand, transient dynamics plays an important role in a number of theoretical approaches in the reviewed projects, especially in the integrations-throughtransients generalization of mode-coupling theory, and has proven useful also in experiments, in e.g., micro- and macro-rheology. It can also be studied under electric bias in dielectrics, in computer simulations, and in single-particle hopping models. Start-up flow (or cessation-flow) through a channel depends on the driving, and generically becomes inhomogeneous with time. The transients thus shed light on the mechanisms of relaxation and whether they can be captured in terms of specific relaxation entities.

Third, insights result from varying the spatial resolution. Micro-rheology considers a single probe particle immersed in a highly complex environment. It provides very local information on the material. The surrounding complex fluid is structured on comparable length scales as the probe. It exhibits slow and spectrally broad dynamics, in particular, close to the glass transition and when the region surrounding the probe is melted locally. The coupling of the probe to its environment is an interesting topic in statistical physics in itself, it is an open non-equilibrium system, but the very large noise observed at low temperatures is also relevant to the mechanical behavior of metallic glasses used in technological devices far below their glass transition temperature. Varying the spatial scale over which external fields are applied and the responses are observed provides information on the behavior of the static and dynamic correlation lengths at the glass transition which are investigated intensely.

A number of research topics and specific questions unite the projects reported in this special issue.

The Breakdown of linear response is studied. Analysis of the nonlinear response necessarily also involves understanding of the breakdown of linear response. Therefore all manuscripts collected here relate to this issue. It has a particular importance for the experimental nonlinear spectroscopy performed using mechanical $[1,2]$ and electrical fields $[6,9]$. The leading higher-harmonic contributions in large amplitude 
oscillatory shearing in the dielectric susceptibility and the conductivity are measured experimentally as a function of the magnitude of the external field, which has to be controlled accurately. As one of the highlights reported here, the structure of the critical clusters at the thermodynamic glass transition could be revealed [9]. The dependence of the breakdown of linear response to the chosen external field variable for the different experimental protocols needs careful consideration from the theoretical perspective as described in reference [3]. Furthermore, in reference [8] the breakdown of linear response when approaching the critical point in Lorentz gases is monitored, revealing where the impact of increasing correlation lengths can be studied. In the framework of the potential energy landscape analysis, the breakdown of linear response in sheared supercooled liquids is related to a reduction of energy barriers or to an increase of the effective temperature [4].

Transient response of supercooled liquids to large quenches of external fields. Large and rapid changes in external mechanical and electric fields allow to monitor the resulting slow evolution of stresses or strain fields, and charge and density distributions in supercooled and granular fluids. In reference [1], stresses are applied macroscopically to metallic alloys ('creep under load'), while reference [2] describes the behavior of mixtures of colloids under load. Reference [3] continues with colloidal dispersions from a theoretical point of view, comparing macroscopic creep with theory and simulation of pressure driven flow confined in channels. There a characteristic inhomogeneity in the flow field is the formation of a plug. Micro-rheology in the constant-force mode observes an individual probe particle, which is pulled constantly. Experimental and theoretical insights thereby gained for granular media are reported in reference [5]. The motion of an intruder in a two-dimensional dense granular fluid is analyzed, which is also addressed using a continuous-time random-walk analysis and simulations in reference [4] of this issue. Conductivity measurements in ionic liquids under an applied dc field require careful tuning of the experimental parameters in order to prevent electrochemical degrading the sample [6]. The results can be interpreted using computer simulations on ionic transport in model systems of disordered media [7] and simulations of binary charged mixtures [4]. Model systems as well as more chemically realistic systems are discussed.

Length scales of heterogeneous dynamics in nonlinear response. Length scales play important roles in the transport of tracer particles in disordered media. Dynamical heterogeneities exhibit length scales which can only be resolved when considering time-dependent correlation functions. Micro-rheology has turned out sensitive to these heterogeneities $[4,8]$ considering single probes driven through well-characterized models of heterogeneous media or simple particle models. Changing the character of the disorder in the surrounding matrix, and the interactions between mobile particles when their concentration increases, and between particle species of strongly different mobility, the relevant time-dependent correlation functions and susceptibilities are determined in simulations and analytical models. This provides insights into the transport of binary mixtures studied by confocal microscopy [2] and to (transient) conductivity measurements [6]. Micro-rheology in dense granular fluids also records spatial correlations around pulled intruders [5]. Reference [3] reports inhomogeneities which originate from the boundary conditions of flows through confinements. The effects of nonlocal terms in constitutive equations are tested comparing to simulations. Finite-size effects also provide information about the length scales as described in reference [4].

The manuscripts collected in this special issue report on experimental, simulational, and theoretical results obtained on a number of model glass-forming systems including colloidal dispersions, metallic alloys, ionic and molecular liquids. The investigations use mechanical and electric fields to probe the nonlinear response in vitrifying systems. 
Riechers and Samwer report on the nonlinear mechanical response of bulk metallic glasses [1]. The deformation behavior under large amplitude oscillatory stress of a ternary alloy is investigated slightly below is calorimetric glass transition. Two different analyses of the nonlinear strains are performed in order to study the importance of the activation and correlation of local microstructural rearrangements. A detailed analysis of strain increments reveals power-law behavior in the strain distributions, which is indicative of avalanche-like accumulation of plastic events even at stresses well below the yield stress. Sentjabrskaja et al. investigate colloidal dispersions where microscopy reveals individual particle trajectories and the local motion can be related to the macroscopic stress vs strain relation [2]. Mixing effects, crucial for the preparation of bulk metallic glasses, are also important in colloidal dispersions. Binary mixture are studied in a wide region of parameters, revealing a number of different frozen-in states. Also, the response under different applied external drivings is studied, shear-rate controlled vs stress-controlled, and differences in the transient approach to the stationary state are related to the microscopic particle motion and to (intermediate) heterogeneous structural dynamics. The link between the (overdamped) motion of individual particles and the macroscopic mechanical response is picked up from the view point of theory in the report by Cárdenas et al. [3]. Motion enforced by large forces, yielding under static load, and pressure-driven inhomogeneous flow through narrow channels are studied. Constitutive equations relating stress and strain locally are derived from the microscopic mode-coupling theory in the integrations-through-transients formalism and employed together with Lattice-Boltzmann simulations solving for the flow fields. While in the reported colloidal systems excluded volume interactions dominate, in molecular glass formers the potential-energy landscape can be used to understand the structural dynamics. The report by Heuer and coworkers describes the motion of mechanically driven glass-forming systems from the perspective of the potentialenergy landscape [4]. The variation of relaxation times is explained by general concepts like that of an effective temperature, which is already encountered in the solution of a 1D generalized Langevin equation. Furthermore, the (nonlinear) conductivity of charged systems is related to the ability of pair formation and the absence of structural modifications, even for very strong fields. In granular systems, thermal energy is irrelevant while the external drive supplies energy to balance the dissipation arising from inelastic collisions and also causes the noise. Sperl and Zippelius report on studies of the glass transition in granular fluids combining mode-coupling theory and active micro-rheology experiments [5]. They find that the theory can be generalized to granular states far from equilibrium and identify universal and granular-specific features. Especially the velocity-force relation measured in micro-rheology can be analyzed in detail. Motion of tagged particles in disordered media lies in the center of the next two reports also. Horbach and coworkers report on particle-based computer simulations of simple models suggested for the description of anomalous transport in heterogeneous media [7]. They find sub-diffusive as well as super-diffusive behavior for intermediate times depending on the interactions and explain how simple models can be mapped onto more complex situations like motion in glass formers. Recent progress in analytical approaches to determine asymptotic laws for anomalous motion or non-analytic field dependences is summarized in the review by Mandal et al. [8]. The analysis of some of the models simulated can be performed rigorously, e.g., for low densities or close to a percolation transition. A rather universal picture emerges for the leading nonlinear-force corrections which generically enter non-analytically. Glass transitions in confinement are also analyzed extending mode-coupling theory, which adds to the picture about anomalous transport in confined systems. Nonlinear conductivities, already considered by theoretical approaches in reference [4], are picked up in the the report by Roling and coworkers from the experimental perspective [6]. Comparing to the well-understood phenomena in diluted electrolyte solutions, novel investigations 
of the nonlinear ionic conductivity of supercooled ionic liquids are presented and discussed. Large nonlinear effects at the glass transition are observed which cannot be rationalized by the theories developed for diluted electrolytes. Rather the understanding starting with approaches from supercooled liquids [4] needs to be extended. Nonlinear effects in dielectric spectra have become accessible experimentally only within the last few years. Lunkenheimer, Loidl, and colleagues report on the most recent advances including to measure the fifth-order response [9]. Their broad-band investigations of the leading nonlinear correction at the third harmonic provide insights into the classification of glass-forming systems: Strong versus fragile, type A or B depending on the existence of relaxation processes on intermediate frequency ranges, monohydroxy alcohols with specific molecular relaxation processes, and plastic crystals. In all cases, the nonlinear response provides insights into the glass-forming characteristics of the different classes. Most recently, the measurement of the group of the next-to leading nonlinear correction at the fifth harmonic has been interpreted in terms of an equilibrium phase transition and clearly suggests the value to extend the nonlinear spectroscopy and its theoretical interpretation.

The reported experimental, theoretical, and simulational approaches to the nonlinear response in supercooled melts and glasses focus on fundamental aspects. Techniques are being developed to understand from first principles the response of cooperative degrees of freedom driven far from equilibrium by external perturbations and because of the intrinsic loss of ergodicity. The technological processing of melts and glasses and the tuning of their final properties, however, also uses external fields in great variation. Frozen-in stress-networks, which determine the mechanical properties of glass-panels, are just one example, which has huge technological significance. A colloidal model system was established to investigate the frozen-in stresses quantitatively and to observe the local particle motions during their arrest. The long-term behavior of amorphous solids under constant or cyclic load is another key aspect for the deployment of e.g., bulk metallic glasses. The investigations of the transient response reported here will contribute to their fundamental description, and, in the long term, to their controlled design. Charge transport in glassy ionic liquids in batteries and general electrochemical devices is another technologically important aspect where the reported results contribute to establishing a fundamental understanding.

Discussions with all members of the research unit are gratefully acknowledged. Financial support was provided by the Deutsche Forschungsgemeinschaft (DFG) through FOR 1394.

\section{References}

1. B. Riechers, K. Samwer, Eur. Phys. J. Special Topics 226, 2997 (2017)

2. T. Sentjabrskaja, M. Laurati, S. Egelhaaf, Eur. Phys. J. Special Topics 226, 3023 (2017)

3. H. Cárdenas, F. Frahsa, S. Fritschi, A. Nicolas, S. Papenkort, T. Voigtmann, M. Fuchs, Eur. Phys. J. Special Topics 226, 3039 (2017)

4. A. Heuer, C.F. Schroer, D. Diddens, C. Rehwald, M. Blank-Burian, Eur. Phys. J. Special Topics 226, 3061 (2017)

5. M. Sperl, A. Zippelius, Eur. Phys. J. Special Topics 226, 3079 (2017)

6. B. Roling, L.N. Patro, O. Burghaus, M. Graf, Eur. Phys. J. Special Topics 226, 3095 (2017)

7. J. Horbach, N.H. Siboni, S.K. Schnyder, Eur. Phys. J. Special Topics 226, 3113 (2017)

8. S. Mandal, M. Spanner-Denzer, S. Leitmann, T. Franosch, Eur. Phys. J. Special Topics 226, 3129 (2017)

9. P. Lunkenheimer, M. Michl, T. Bauer, A. Loidl, Eur. Phys. J. Special Topics 226, 3157 (2017) 